\section{Heterocyclic Chemistry}

\section{by Irina P. Beletskaya}

The XXI European Colloquium on Heterocyclic Chemistry $(E C H C)$ was held 12-15 September 2004 in Sopron, Hungary. The conference, which was organized by the Chemical Research Center of Hungarian Academy of Sciences and the Hungarian Chemical Society, drew over 300 participants, including 100 young chemists from 24 countries. The program included 12 invited lectures and 180 posters, of which 12 were selected for oral presentation. The conference chairman Gyorgy Hajos and co-chairman Peter Matyus helped create a highly organized event with a very creative and friendly atmosphere.

The chemistry of heterocyclic compounds comprises a very broad field as is clearly seen from this partial list of invited lectures:

- F. Diederich (Switzerland), "Heterocycles in the design of nonpeptidic enzyme inhibitors"

- I.P. Beletskaya (Russia), "Transition metal catalysis in heterocyclic chemistry"

- J. Alvarez-Builla (Spain), "Pd-reactions in charged heterocyclic species"

- G. Keglevich (Hungary), "P-Heterocyclic chemistry"

- K.R. Seddon (Ireland), "Heterocyclic cations for ionic liquids"

- J.A. Gladysz (Germany), "Design of molecular devises ("rotors", "gyroscopes") using alkene and alkyne metathesis reactions"

- I.E. Marco (Belgium), "Tandem pericyclic reactions of 2-pyrone derivatives"

- J.M. Bakke (Norway), "Synthesis of nitropyridines using $\mathrm{N}_{2} \mathrm{O}_{5} / \mathrm{SO}_{2}$ "

- N. Haider (Austria), "Cycloaddition routes to condensed carbazoles"

- P.J. Dunn (Pfizer), "The history of the discovery of Viagra and other PDE 5 inhibitors"

- K. Hideg (Hungary), "The chemistry and biology of heterocyclic nitroxide radicals"

- S. Florio (Italy), "Utilization of oxiranyllithiums in various interesting asymmetric syntheses"

The conference demonstrated that innovative methods of modern chemistry (new synthetic methodologies, new reaction media, novel catalytic methods, metal-catalyzed and metal-mediated processes, new physical methods of activation [microwave, for instance]) are widely used in the chemistry of heterocyclic compounds. The potential of this chemistry for the synthesis of new and useful compounds is truly inexhaustible.

The next Heterocyclic Colloquium will be held in Italy in 2006.

Irina P. Beletskaya <beletska@org.chem.msu.su> is a professor in the Department of Chemistry at Moscow State University. She served as IUPAC representative at the ECHC and is also a former president of the Organic and Biomolecular Chemistry Division of IUPAC.

\section{Soil Science \\ by Qiaoyun Huang}

The 4th International Symposium on Interactions of Soil Minerals with Organic Components and Microorganisms (ISMOM2004) was held 20-23 September 2004 at the Huazhong Agricultural University in Wuhan, China. The conference was sponsored by the International Union of Soil Sciences (IUSS) and IUPAC. The meeting attracted 135 delegates from 21 countries. The theme of ISMOM2004 was the "Environmental Significance of MineralOrganic Component-Microorganism Interactions in Terrestrial Systems." The conference program was divided into the following six sessions:

- Transformation and Dynamics of Pollutants in Soil Environments

- Chemical, Biological and Biochemical Processes in the Rhizosphere

- Bioavailability of Metals, Nonmetals and Xenobiotics Immobilized on Soil Components

- Distribution and Activity of Biomolecules in Terrestrial Systems

- Interactions between Soil Microbial Biomass and Organic Matter/Nutrient Transformations

- Impact of Interactions among Soil Mineral Colloids, Organic Matter and Biota on Risk Assessment and Restoration of Terrestrial Ecosystems

All sessions consisted of oral and poster presentations. There were 2 plenary lectures, 9 invited speakers, 36 oral presentations and 45 posters. Nicola Senesi, from the University of Bari, Italy, presented an IUPAC lecture on "Metal-Humic Substance Complexes in Soil." Pan Ming Huang from the University of Saskatchewan, Canada, gave a plenary lecture on 